\title{
Corela
}

Cognition, représentation, langage

$1-2 \mid 2003$

Vol. $1, \mathrm{n}^{\circ} 2$

\section{Un autre regard sur la répartition des rôles entre as et like dans la construction de la comparaison}

\section{Emmanuelle Roussel}

\section{OpenEdition}

\section{Journals}

Édition électronique

URL : http://journals.openedition.org/corela/705

DOI : $10.4000 /$ corela.705

ISSN : 1638-573X

Éditeur

Cercle linguistique du Centre et de l'Ouest - CerLICO

Référence électronique

Emmanuelle Roussel, « Un autre regard sur la répartition des rôles entre as et like dans la construction de la comparaison », Corela [En ligne], 1-2 | 2003, mis en ligne le 01 décembre 2003, consulté le 20 avril 2019. URL : http://journals.openedition.org/corela/705 ; DOI : 10.4000/corela.705

Ce document a été généré automatiquement le 20 avril 2019

\section{(c) (1) (3) (-)}

Corela - cognition, représentation, langage est mis à disposition selon les termes de la licence Creative Commons Attribution - Pas d'Utilisation Commerciale - Partage dans les Mêmes Conditions 4.0 International. 


\title{
Un autre regard sur la répartition des rôles entre as et like dans la construction de la comparaison
}

\author{
Emmanuelle Roussel
}

1 L'étude que je propose ici se fonde sur l'observation et l'analyse du fonctionnement de as et like dans les constructions où il est question de l'expression d'une comparaison construite sur un repère de nature nominale (du type: ... as / like dét. $\mathrm{N}^{1}$ ). Les deux opérateurs sont alors prépositions ${ }^{2}$.

2 Mon objet n'est pas ici de remettre en question ce qui a été dit à ce sujet, l'identification et la différenciation que mettent en place les deux opérateurs étant maintenant acquises, et ce, même si je suis amenée à en parler à nouveau. Le sens de mon travail est ailleurs ; je souhaite réfléchir autrement à la question en essayant de montrer, d'une part, la manière dont se construit le phénomène à la base de celui d'identification et de celui de différenciation et, d'autre part, la similitude qui existe entre ce que nous pourrions appeler le "système as / like" et d'autres systèmes déjà existants dans la langue.

Revenons tout d'abord sur le concept même de comparaison. La définition que nous fournit le Petit Larousse est la suivante :

Action de comparer, de noter les ressemblances et les dissemblances entre deux ou plusieurs personnes ou choses ; parallèle."

Cette action de comparer est elle-même présentée ainsi :

1. Rapprocher deux ou plusieurs objets pour en établir les ressemblances et les différences [...]. 2. Souligner, par une comparaison, les mérites respectifs de deux ou plusieurs choses ou personnes. 3. Faire valoir une ressemblance, une analogie entre deux êtres ou deux choses.

Nous y voyons deux points intéressants.

6 En premier lieu, le principe même de la comparaison apparaît comme étant relativement simple, la définition proposée par le dictionnaire correspondant tout à fait à toutes celles qui ont pu être proposées d'un point de vue strictement linguistique ${ }^{3}$. En fait, il s'agit de 
confronter deux (ou plus) entités. Cette confrontation doit en outre faire gagner en référence les entités en question. La comparaison doit en effet apporter un surplus sur le plan de la référence, dans la mesure où son but est de permettre une meilleure délimitation des référents. Ce surplus est d'ordre informationnel. Le procédé de la comparaison apporte davantage d'informations sur une entité. Puisque l'on travaille sur un apport d'informations, le principe fonctionne à l'évidence lorsque la comparaison est une comparaison d'infériorité. Ce n'est pas tant en effet la quantité référentielle de l'entité qu'il s'agit de mesurer, que la quantité informationnelle portant sur cette entité, qui lui fait par conséquent gagner en qualité notionnelle ${ }^{4}$.

7 En second lieu, l'observation des termes employés est révélatrice, notamment dans la seconde définition. Il s'agit toujours de termes positifs (" ressemblances", "parallèle", " rapprocher", " mérites", " faire valoir", " analogie "). Nous sommes donc bien dans un procédé de valuation, la comparaison vise à améliorer, à enrichir notionnellement le référent de l'entité repérée en s'appuyant sur les propriétés de l'entité repère.

En français, l'opérateur communément associé à la comparaison est “comme". Il peut signifier "tel que " et sert précisément à exprimer la comparaison au sens strict. Il peut encore être utilisé dans le type de phrase suivante: "ça s'est passé comme il voulait", dans laquelle il traduit plus précisément cette fois la manière. Nous laissons de côté ce sens de "comme". En poursuivant les recherches le concernant dans sa première acceptation (= " tel que "), on trouve dans le Larousse de Poche qu'il :

s'emploie pour exprimer une comparaison d'égalité, de même que " aussi... que " et

" autant... que ". (Larousse de Poche : 391)

9 En étendant la recherche, on s'aperçoit qu'il est apparu dans les tours suivants, avec les valeurs ainsi décrites :

“Aussi ... comme”, “ autant ... comme”, usuels autrefois, ne s'emploient plus que dans l'usage populaire et dans certaines provinces : * Il y a autant d'inconvénients comme d'avantages".

[...]

"Comme qui dirait" indique dans la langue familière une analogie ( = "pour ainsi dire, une sorte de"), parfois avec une nuance plaisante: "c'est une grande place, comme qui dirait la place de la Concorde à Paris". Il y a comme qui dirait une difficulté."

"Comme qui dirait que " + indicatif introduit, dans la langue très familière et sur le mode plaisant, une réflexion suscitée par une observation ${ }^{5}$ : "Comme qui dirait que j'arrive trop tard ! " (Le Petit Robert : 1681).

10 Ce passage par le français est intéressant pour notre propos à double titre.

11 D'une part, on y retrouve l'étiquette de "populaire ", "langue familière " qui est souvent apposée à like dans ses emplois dits “impropres” de conjonction. L'anglais et le français ont un parcours similaire en ce qui concerne l'expression de la comparaison: ils disposent d'une expression formelle et d'une autre qui l'est moins. De fait, on retrouve des traces de mauvais usage assez tôt en anglais. Like, à l'époque de Shakespeare, succède à like as, dans le champ informel, là où as est plus littéraire. Si la répartition des champs d'emploi se dessine alors, il n'en demeure pas moins que les deux opérateurs se trouvaient auparavant liés, constituaient une seule et même entité. Il y a donc eu une époque où leur fusion n'était aucunement problématique.

D'autre part, j'ai souligné dans la dernière citation un point qui va se révéler très pertinent dans la suite de ce travail. Le critère de l'observation semble en effet être un élément de poids dans la sélection de like ${ }^{6}$. C'est, entre autres, ce que j'aimerais montrer. 
On retrouverait une similitude de comportement entre le français et l'anglais dans l'élaboration de la comparaison, prioritairement peut-être à une correspondance terme à terme.

Nous nous trouvons donc face à l'équation suivante aujourd'hui : un terme en français pour deux termes en anglais. La question à laquelle je souhaiterais apporter quelques éléments de réponse est donc celle-ci : qu'est-ce qui fait que l'anglais a su / pu continuer à départager ses deux opérateurs dans leurs emplois de prépositions? Qu'ont-ils de si particulier?

\section{Observation}

Je vais, dans cette première partie, examiner ce que l'on peut trouver sur as et like dans les emplois qui m'intéressent.

\subsection{Etymologie}

L'étymologie de as et de like est connue. Je me contenterai plus loin de la rappeler. Ce qui m'intéressait davantage dans mon étude était de recenser les divers emplois de termes relatifs à la comparaison et construits sur as et like, pour éventuellement tenter d'en dégager une systématique susceptible d'éclairer le fonctionnement actuel des deux prépositions.

que j'ai pu trouver est intéressant. On rencontre aujourd'hui des choses comme as like as two peas, as like as not où les deux opérateurs sont réunis. D'où une première conclusion. Autant as like as two peas et as like as not sont admis ${ }^{7}$, autant * like as like I did, par exemple, ne l'est pas, ni † like as a nightingale, catalogué comme archaïque ${ }^{8}$. Les opérateurs ne sont donc pas toujours interchangeables.

Dans le même ordre d'idée, on dispose de likely, likewise, unlike ${ }^{9}$ mais pas de * asly, * aswise ou * unas. Autre argument en faveur de la spécialisation des emplois de like et de as, respectivement. On dispose également de except / but as mais pas de * except / but like ${ }^{10} \hat{\imath}$ Pour ce qui est de unlike, il est glosable par not like ${ }^{11}$. Si je m'en tiens pour l'instant à ce qui a été dit au sujet de as et like, à savoir as est un opérateur d'identification et like un opérateur de différenciation dans leur construction de la comparaison, cela signifierait que dans le couple unlike / * unas, l'acceptabilité du premier indiquerait que l'on peut nier la similarité, la ressemblance (induite par like), mais pas l'identité (induite par as). Cela ne me semble pas un argument suffisant. Sachant en outre que not as existe bel et bien. Ces choses méritent d'être creusées. La symétrie pure entre as et like n'existe pas. J'y reviendrai.

18 J'ai par la suite, dans un souci d'exhaustivité, cherché à savoir si alike vient de la combinaison de as + like, selon un critère strictement formel. De deux choses l'une : si tel est le cas, cela pourrait indiquer la raison de leur utilisation dans le même champ ; dans le cas contraire, et c'est effectivement ce qui se passe, il devient plus que jamais impératif d'approfondir les raisons de leur emploi respectif.

19 A l'évidence, alike ne provient pas de as + like. Il est issu de ge + like (ge-lice), à savoir ge + ‘ having the same character or quality' (= lice), qui par la suite donnera like $e^{12}$.

Ge-lice date du Vieil-Anglais (449-1100), et signifie littéralement, on le sait, " avec "-“ forme, corps ", c'est-à-dire " ayant le corps ou la forme de ". Il a donné ilich-e / 
ilik-e au début du Moyen-Anglais (1100-1400), qui devient alike vers 1500. Parallèlement se développe like préposition à partir du XIIe siècle, puis like verbe et enfin like suffixe (fin $\mathrm{XVe})^{13}$.

21 Alike équivaut à 'like one another', il commence par être employé comme adjectif puis comme adverbe. Like préposition se glose en anglais 'having the same qualities, feautres or characteristics' ou 'having the body or shape in common with one another' ${ }^{14}$, qui fait peut-être mieux ressortir sa parenté avec alike.

Ce qui, historiquement parlant, signifie que like est une forme tronquée de alike, et corrobore le fait que le préfixe $a$ - ne vient pas de as, il est autonome. On en retiendra que alike est uniquement lié à like, et que cela peut contribuer à expliquer que as et like ne sont pas synonymes à $100 \%$. Chacun a ses caractéristiques propres.

Maintenant as. As signifie 'to that or such a degree', c'est-à-dire 'in the manner or to the extent in which'. Il provient de ase, als, allomorphes de alse, lui-même issu du Vieil-Anglais alswâ, ealswâa (qui signifie 'wholly so, quite in that way'), à savoir also. Also n'est autre que all + so. Le parcours total de alswâ est le suivant :

Alswâ alse forme faible als as ${ }^{15}$

? also ? forme forte ase (disparue)

Nous voyons bien d'une part que as a pour origine also. J'ai également trouvé que le as du Moyen-Anglais est présenté comme étant le vestige d'une structure corrélative construite sur ealswa $\hat{a}^{16}$. La construction en as ... as est un des emplois possibles de as en MoyenAnglais. L'origine des comparatifs d'égalité remonte donc loin.

Il résulte de tout ce qui précède que as et like se sont développés au même moment de l'histoire de la langue anglaise, mais qu'ils n'ont rien en commun ni dans leur origine ni dans leur sémantisme, contrairement aux apparences. Leur orthographe actuelle date du début de l'époque du Moyen-Anglais, du XIIe siècle précisément.

Voyons à présent quelles sont leurs caractéristiques d'emploi en anglais contemporain.

\subsection{Faits}

27 Le premier point auquel je souhaiterais m'intéresser concerne ce comportement alterné, qui n'est du reste pas un cas isolé dans la langue anglaise, de as et like en préposition ou conjonction.

On nous dit dans les grammaires que la différence d'emploi de as et like s'explique selon la nature de ce qui les suit. Dans la langue formelle donc, as et like ont en commun de pouvoir tous deux introduire un syntagme prépositionnel et, dans le même temps, ils ont chacun pour propriété d'introduire une proposition ou un syntagme nominal pour as et un syntagme nominal pour like. D'où la répartition suivante :

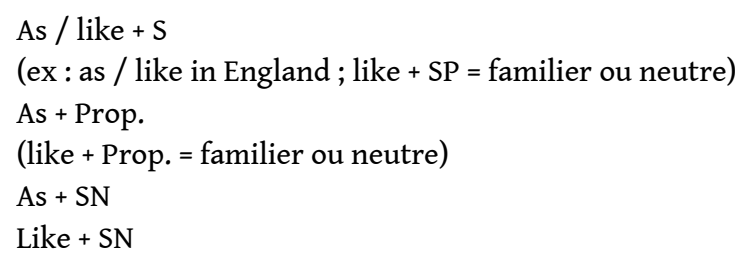

29 Autrement dit, as et like se rapprochent lorsqu'ils sont conjonction, la différence qui subsiste ne se situant qu'au niveau de langue, et ils s'opposent sémantiquement dès lors 
qu'ils sont préposition, as signifiant “ en tant que " (as a teacher : en tant qu'enseignant) ${ }^{17}$ et like, " comme" (like a teacher : " comme un enseignant ").

\subsubsection{Travaux} pu consulter. Après avoir mentionné que as if est remplacé par like en anglais américain ${ }^{18}$, Quirk remarque que as et like (pour ce qui est de l'expression de la comparaison et non de celle de la manière) expriment tous deux la comparaison, et peuvent pareillement être introduits par just ou exactly (Quirk et al. 1985 : 1110).

31 Il note également que as ... as dénote "la comparaison de l'équivalence" (Quirk et al.: 1137), que like est apte à renvoyer à la ressemblance physique :

(8) The man like John is over there.

Et à la ressemblance comportementale :

(9) A man like John would never do that.

Il est vain de souligner que bien d'autres facteurs que as et like dans ces énoncés contribuent à leur sémantisme final (cf. le rôle des déterminants et du modal notamment). Force est de constater que ces remarques ne sont pas d'une grande utilité pour qui cherche à comprendre les phénomènes en jeu.

On trouve des remarques plus fouillées chez Lapaire et Rotgé. Ainsi :

As comparatif est glosé, entre autres, par in like manner. (Lapaire et Rotgé 1991:

259).

Dans les " comparatifs d'égalité ", la similitude est déclarée au nom d'un concept précisé dans le contexte : apparence, aptitude, qualité, etc. (1991:260)

La similitude portée par like, contrairement à ce qu'elle peut induire chez as, ne mène pas à l'identité. Like ne franchit pas les limites de l'analogie (qui est correspondance - ressemblance entre deux termes essentiellement différents). (1991 : 263)

[...]

Même lorsque l'interchangeabilité est parfaite, une différence de stratégie existe toujours. As, estimons-nous, est plus actualisateur [...] et fusionnel que like, moins assertif et plus séparateur. (1991: 263)

Je souhaiterais également évoquer l'article de Lab, dans lequel sont abordées les notions d'identification et de différenciation du point de vue de la Théorie des Opérations Enonciatives ${ }^{19}$. Comme je l'ai dit plus haut, il n'est pas dans mon propos de remettre en question ces principes. Si la matière est la même, il en est fait ici un usage différent (... as / like $+\mathrm{N}$ ). A présent que les origines des marqueurs a été dégagée, je propose d'aller audelà de ces concepts d'identification et de différenciation. Dans l'article en question, on peut trouver des pistes pertinentes pour mon propos. Ainsi Lab (1999: 90) cite une définition de like que je souhaiterais souligner car elle viendra plus tard illustrer l'objet de ce travail :

In spoken English, people sometimes say like when they are hesitating or when they are thinking about what to say next. This is a very informal use, which many speakers of English consider to be incorrect. (Collins Cobuild)

On remarquera pour l'instant que l'on se situe là au niveau de l'observation d'un usage, mais que l'on n'explique pas cet usage. J'en retiens le concept d'hésitation, qui me paraît important. De même, on peut lire, toujours au sujet de like (Lab, ibid.) : 
Rappelons tout d'abord que l'opération sous-jacente à like est une opération de DIFFERENCIATION, c'est-à-dire de mise en relation de deux termes avec MAINTIEN DE L'ALTERITE, d'où la DISTANCE préservée entre ces deux termes. Or cette distance peut être présentée comme une distance à parcourir: qu'elle soit parcourue avec succès (et like s'efface alors éventuellement devant as, [...]) ou que, comme ici, elle soit présentée comme difficile, voire impossible à parcourir.

Ce que je me demande c'est si, dans le sémantisme de like, il est réellement souhaitable de parcourir une quelconque distance, aussi notionnelle soit-elle. Je pencherais davantage pour une problématique centrée autour de la perception de l'énonciateur, mentionnée dans l'article, même si je la conçois différemment :

[...], et avec like de façon générale, il y a prise en charge explicite du contenu propositionnel par un sujet. Nous dirons qu'avec like l'énonciateur “active" le sujet en tant que sujet percevant, à l'origine de la perception. (Lab : 99) 20

Ce point de vue de la perception est central dans mon étude. L'analyse de mon corpus montre en effet qu'il est possible de dégager un classement des occurrences de as et de like illustrant une reprise par l'énonciateur de critères catégorisants déjà existants pour as, et une participation active de l'énonciateur dans l'élaboration du processus de comparaison pour like.

\subsubsection{Répartition de as et like}

Les énoncés en as se répartissent généralement selon que le nom repère constitue une entité répertoriée, ou un sous-groupe (s'appuyant sur un groupe existant) créé pour les besoins de l'énoncé. Ceux en like ont en commun de refléter une construction du repère pour / au moment de l'énoncé. Les moyens utilisés sont à l'évidence différents, mais le principe est toujours le même.

Ainsi, concernant as, j'ai relevé des énoncés comme :

(10) 'Ah!' sang Jenny, high and pure as the sea maiden of the Hebrides whom Sandy had been talking about . (Spark : 23)

(11) (As) they awaited for the tram-car Miss Brodie said, 'I had lodgings in this street when I first came to Edinburgh as a student. (Spark : 40)

41 Où $a$ student fonctionne comme entité stabilisée en tant que sous-groupe.

(12) But so far, they had not engaged her attention as men, she knew them only as supporters, and was proudly grateful. (Spark : 48)

Cette fois, il y a passage d'un premier sous-groupe (men) à un second (supporters).

(13) When they conferred amongst themselves on the subject they had to admit, at last, and without doubt, that she was really an exciting woman as a woman. (Spark : 116)

A nouveau, il est fait référence à woman comme entité stabilisée représentant un sousgroupe. Il en est de même de a feature of vowels dans (14) ci-dessous :

(14) The second [dimension] is 'nazalisation'. English disregards this as a feature of vowels. (Bolinger : 27)

(15) It is often hard to decide whether a lengthened sound ought to be regarded as a single sound with extra length, or as two identical sounds side by side. (Bolinger : 27)

Ici, nous observons la création de deux sous-groupes repères. Parallèlement se pose la question de savoir pourquoi, dans cette construction verbale répertoriée to (dis)regard something / someone as, c'est as qui a été choisi. La raison en est sans doute qu'elle s'appuie 
nécessairement sur un acquis. Or, nous le verrons, seul as peut l'introduire. Le phénomène se retrouve avec d'autres verbes :

(16) Miss Mackay said to Sandy confidentially when her turn came round - because she treated the older girls as equals, which is to say, as equals definitely wearing uniforms - '[...]'. (Spark : 115)

Et nous verrons plus loin qu'on le rencontre à nouveau dès lors que le repère constitue une entité répertoriée (dans le dictionnaire).

Terminons ce premier aperçu avec quatre autres énoncés :

(17) Probably all languages use relative 'height' as a sign of importance. [...]. (Bolinger : 32 )

(18) At the level of 'syntax' we find words again, but now we find them as representatives of sound classes (noun, verb, adjective, and so on) rather than of meanings. (Bolinger : 40)

(19) One process uses words themselves as raw material for new words. (Bolinger :

54)

47 Le dernier énoncé rassemble les deux propriétés de renvoyer à un sous-groupe et à une entité répertoriée. Il est vrai que la frontière entre les deux critères est mince. Ce phénomène de chevauchement n'est pas rare :

(20) [...] hearers react to the complex sound as a unit. (Bolinger : 41)

Cette fois, non seulement le repère fonctionne comme sous-groupe en ce sens qu'il renvoie à un item catégorisant, mais il fonctionne également comme entité répertoriée (dans le dictionnaire et dans l'usage) dans la mesure où l'unité constitue un critère reconnu d'évaluation. Se comportent de la même manière les énoncés suivants :

(21) And although these concerns at Rose Stanley's eleventh year marked her as a tomboy, they did not go deep into her feminity and it was her superficial knowledge of these topics alone, [...], which stood her in good stead a few years later with the boys. (Spark : 28)

Le fait que la comparaison repose ici sur une entité répertoriée permet la concision du référent du repère. Un seul nom suffit à indiquer de quoi il s'agit, sur quoi se base la comparaison. Il n'est nullement besoin d'autre complémentation.

(22) As with geological formations, the visible mass of linguistic structure forms itself into layers. (Bolinger : 38 )

La construction du repère peut également s'opérer sur une réalité physique avérée, non susceptible d'être remise en cause (22). Il en est de même en (23) et en (24), où la réalitécanon, toujours stabilisée, est définie par un terme technique, appartenant à un lexique spécialisé :

(23) While it is true that the notes in a chord are heard simultaneously, sometimes chords appear as arpeggios, in which the notes are played one after the other. (Bolinger : 42)

(24) [...], no matter how strong the other reasons may be for regarding two phones as allophones of a single phoneme. (Bolinger : 44)

51 En (25), le repère est une citation empruntée à quelqu'un. Elle a donc été au moins une fois réalisée dans le discours :

(25) Instead of saying [...], they prefer to view it as "represented directly by the features which make it up". (Bolinger : 45) ${ }^{21}$

52 Pour ce qui est de (26), la comparaison se base sur des lexies, lexicalisées donc et s'agissant de l'exemple, répertoriées : 
(26) It [English] has syllables consisting of a nucleus alone, as in the words oh and ah, and syllables in which the nucleus is flanked by two or more satellites on each side, as in splashed and sprints. (Bolinger : 47$)^{22}$

Je terminerai par la mention de deux énoncés construits sur une suite $\mathrm{V}$ as $+\mathrm{N}$, qui ressemblent en ce sens à (14), (15) et (16), mais pour lesquels le repère est bien une entité répertoriée :

(27) Most instruments, [...], when played at a given pitch produce not only that pitch, known as the fundamental, but an infinite series of higher pitches each an even multiple of the number of vibrations in the fundamental. (Bolinger : 31$)^{23}$

(28) When one man referred to the occupants of flying saucers as "saucerians", he was using the active suffix -ian. (Bolinger : 54 )

Nous retrouvons toujours le même processus, sur lequel se construisent les énoncés. To refer to something / someone as équivaut à to define. "Définir" implique dans un premier temps (re)connaissance de la chose. A savoir son identification. En outre, dans cet exemple, la définition s'appuie sur un procédé de dérivation productif, donc, partant, existant et entériné.

Dans chacun de ces cas, la structure ne supporte pas like. Pour les raisons invoquées cidessus et celles qui vont être développées dans les lignes qui viennent.

Les énoncés en like n'obéissent pas aux mêmes règles de distribution que les énoncés en as . Loin de s'élaborer sur des repères ayant la particularité de créer un ou des sous-groupe (s) discriminants, ils mettent en évidence tout un travail de délimitation du repère. Celuici est, à l'inverse de ce qui se passe avec as, en édification, en phase de constitution.

Le phénomène se révèle dans les exemples suivants :

(29) 'I don't think they did anything like that,', said Sandy. (Spark : 20)

(30) I can't have my girls going up and down to the science room like this. (Spark : 25)

(31) It was her habit to come to me every morning to ask what I would have for breakfast, and she spoke like this: "Wud ye have a red herrin ? - no ye wudn't. [...]. (Spark : 40)

(32) We actually do communicate analogically in situations like this, not using words but holding up our hand to the desired height. (Bolinger : 17)

(33) List two other opposing series like these. (Bolinger : 20)

Le déictique a à chaque fois une valeur fortement anaphorisante (sauf en (31) où elle est cataphorisante) qui, dans le même temps, devient particularisante. Les intervenants savent ce à quoi ils réfèrent, car la chose en question naît ou est née de la situation. On est dans de l'implicite qui par la force des choses devient de l'explicite. Plutôt que de parler d'un mouvement du général au particulier, je préfère à présent parler d'un mouvement de "l'ensemble à l'échantillon". En effet, that, this, these illustrent un cas de ce qu'ils reprennent.

9 En (31) par exemple, on observe un redéploiement en mots de ce à quoi renvoie this, une analyse de ce que this synthétise. On peut bien sûr parler de manière ici, mais la manière est rarement dissociable à $100 \%$ de la comparaison. Et je ne pense pas que cela vienne contredire mon étude.

En (32) pareillement, this peut être interprété comme renvoyant à une manière commune d'effectuer deux relations prédicatives distinctes, tout comme il peut être interprété comme signifiant this one, c'est-à-dire comme pronom déictique extrayant une occurrence de situations en fait. Et alors, on s'aperçoit que ce qui suit like n'est autre qu'une sorte de paraphrase formelle de ce à quoi renvoie situations. De l'ensemble ( 
situations) l'énoncé va vers l'échantillon (this one). L'analyse vaut pour (33), en dépit d'un contexte pluriel. Il ne s'agit pas d'un pluriel totalisant, mais bien d'un pluriel particularisant, quantifiant deux occurrences parmi toutes les autres. On peut certes m'objecter que ce pluriel crée un sous-groupe et que l'énoncé présente alors des traits propres à ceux en as vus plus haut. Je répondrai par la négative, car le sémantisme attaché à ce à quoi réfère these ne se comprend que par rapport au contexte de l'énoncé, il est interne à l'énoncé. These ne peut, tel quel, renvoyer à une entité classifiante répertoriée.

61 Le mouvement que j'essaie de mettre en évidence se dessine dans d'autres énoncés, non construits sur des déictiques. Ainsi :

(34) The progressive spinsters of Edinburgh did not teach in schools, especially in schools of traditional character like Marcia Blaine's School for Girls. (Spark : 42)

(35) Some assisted in the Scottish Movement; others, like Miss Brodie, called themselves Europeans and Edinburgh a European capital, the city of Hume and Boswell. (Spark : 43)

62 En (35) avec like, Miss Brodie renvoie à ce que Miss Brodie représente, c'est-à-dire son “ enveloppe ", et non pas ce qu'elle est, c'est-à-dire sa "substance ". On notera qui plus est que l'alternative en as est difficilement acceptable ici.

(36) That had been a thought suitable only for the enlivening of a sewing period, but Miss Brodie was entitled to something like a status. (Spark : 72)

63 L'ensemble est ici indiqué par something, dont a status est un exemple.

(37) 'Phrases like "the team spirit" are always employed to cut across individualism, love and personal loyalties,' she said. (Spark : 78)

En (37), les guillemets jouent un rôle bien précis d'introduction d'un exemple de phrase possible parmi tant d'autres (également possibles). Les exemples de ce type sont très nombreux.

65 L'organisation des constituants illustre à chaque fois le mouvement sémantique. C'est-àdire que like introduit un segment qui se trouve en position de focus, parce que l'information qu'il contient apporte un plus sur le plan sémantique. Ce segment participe directement de la construction de la signification globale et finale des énoncés en question. Ils fonctionnent tous comme des icônes de la mise en place du sens. Plus on se dirige vers la droite, plus l'information se développe et plus le message véhiculé par l'énoncé se fait prégnant.

66 Cependant, et ce qui est très intéressant, cette représentation iconique par la structure de l'énoncé de sa construction du sens n'est pas l'apanage des énoncés en like. De la même manière, certains énoncés en as reflètent leur mouvement sémantique, mouvement inverse néanmoins. Nous rencontrons alors des énoncés tels les suivants :

(38) She was looking at the hills as if to see there the first and unbetrayable Miss Brodie, indifferent to criticism as a crag. (Spark : 67)

(16) Miss Mackay said to Sandy confidentially when her turn came round - because she treated the older girls as equals, which is to say, as equals definitely wearing school uniform - '[...]'.

(39) Our habit of viewing it [language] as a "thing " is probably unavoidable, even for the linguist, but in a sense it is false. (Bolinger : 12)

67 Ce à quoi je fais référence est peut-être plus clair dans ce dernier énoncé, dans lequel $a$ "thing", par l'intermédiaire de as, vient étoffer sémantiquement it (lequel tire prioritairement sa substance sémantique deson antécédent language). Alors qu'avec like on va de l'ensemble à l'échantillon en tentant de s'approcher au mieux de la définition 
idéale, avec as l'apport sémantique se fait en termes de redéfinition à partir, nous l'avons vu, d'un support qui en soi est déjà un idéal. A des degrés divers, c'est ce qui se passe en (40) qui reprend le tableau des voyelles de l'anglais :
(40) [i:] as in beat
[i] as in bit
[e] as in bait
$[a ̊]$ as in bet. (Bolinger : 25)

En (41) également :

(41) The unmusical qualities - noises - we have seen as components of certain consonants, for example, the hissing stridency of an [s] or the sudden explosion of a [p]. (Bolinger :30)

De tous ces énoncés se dégage une systématique relevant du mouvement.

\subsection{Mouvement de l'énoncé}

Pour bien mettre en évidence ce principe d'un mouvement propre à tel ou tel énoncé selon qu'il est construit sur as ou like, je vais travailler sur des exemples de Dylan Thomas, célèbre pour sa capacité à se jouer de la langue. A partir de quelques énoncés, je me suis rendue compte que les jeux de mots faits sur like sont différents de ceux faits sur as. Par conséquent, like et as n'engendrent, et ne reflètent pas, le même type de mouvement sémantique. Like a ceci de particulier qu'il recrée des comparaisons, alors que as ne fait qu'en reprendre. Like permet de se livrer à des manipulations de signifiants et de signifiés, de reconstruire des similitudes. Like apporte un véritable élan dynamique à la construction du sens. L'introduction de l'œuvre sélectionnée fournit à elle seule un matériel extrêmement intéressant. On y trouve ainsi les comparaisons suivantes, qui se font écho :

(42) [...] in Ocky Milkman's loft's like a mouse with gloves. (Thomas $1954: 2$ )

(43) coughing like nannygoats. (Thomas : 2)

(44) [It is night] in Dai Bread's bakery flying like black flour. (Thomas : 2)

(45) quiet as a domino. (Thomas : 2 )

(46) [...] blind as a mole [...] or blind as Captain Cat there [...] (Thomas : 1 )

Et l'analyse démontre que tout part de la locution figée as quiet as a mouse qui, en tant que proverbe se fait le relais d'un constat. D. Thomas s'en joue et en (42) crée (avec like) une relation en s'appuyant en filigrane sur an iron fist in a velvet glove,qui n'est autre qu'un second proverbe, formé sur as. (43) a comme point de départ le champ sémantique du bruit, dans lequel se trouvent opposés le silence du premier proverbe au bruit que fait la toux. (43) se trouve alors chargé d'autres sémantismes, ceux de la crédulité et de la ferveur. On connaît le sens figuré de goat (" andouille ")et l'auteur passe de "ouaille " à "andouille" avec une facilité que lui permet la langue. Commentaire et jugement sont présents ici. On ne peut le nier. Et ceux-ci se créent sur, avec like. Pas avec as. As n'est pas utilisé de la même manière. (45) et (46) ne font que reprendre une construction (as quiet as a mouse; as blind as a bat). La construction est la même donc, il y a simplement, pourraiton dire, reprise de "l'enveloppe textuelle". Il y a ici moins de travail énonciatif qu'avec like. On repose sur une identité de construction. La méthode est beaucoup plus statique avec as qu'elle ne peut l'être avec like. On parvient alors sur le système like = dynamique, as = statique, qui a par le passé été rapproché du système have / be. Ce que permet like, c'est bien de reconstruire des similitudes à partir d'identités déjà installées par as (en tant que lexies répertoriées). 

sémantique que je vois apparaître dans les énoncés étudiés. Les énoncés ci-dessous vont pouvoir servir de support :

(47) When four-year-olds are given a sentence like 'I gave the dog the bone' they will repeat it and understand it. (Bolinger : 7)

(48) [...] 4. simple sentence structure, as, for example, avoidance of the passive voice (Bolinger: 4 )

(18) At the level of 'syntax' we find words again, but now we find them as representatives of sound classes (noun, verb, adjective, and so on) rather than of meanings.

(20) [...] hearers react to the complex sound as a unit.

La chose qui s'y passe est la suivante. En (47), le mouvement sémantique de l'énoncé est :

\section{P like Q}

Où $Q$ apporte une enveloppe formelle ainsi qu'un contenu sémantique à $P$. C'est-à-dire que $\mathrm{Q}$ développe le contenu sémantique de $\mathrm{P}$, à des fins de définition. Le mouvement du sens dans l'énoncé est linéaire, il va de la gauche (l'ensemble) vers la droite (l'échantillon ${ }^{24}$ ), siège de l'information nouvelle ou importante. Plus on va vers la droite, plus on gagne en sens, et plus on peut se représenter la comparaison, intellectuellement parlant. D'où très certainement toutes les structures en $V$ like $X$ (sound like X, taste like X, look like X, etc .) :

(49) In ordinary speech 'one's own' sounds like 'one zone', 'an ungodly' like 'a nun godly', 'palisade's like 'palace aides', and so on. (Bolinger : 46) Tout s'agence au fil de l'énonciation.

Le mouvement sémantique de (18) et (20) va dans le sens inverse. On a :

$\mathbf{P}$ as $\mathbf{Q}$ sémantique. $Q$ est une illustration de $P$. La redéfinition de $P$ donnée par $Q$ se fait en lui attribuant une nouvelle identité, identifiée au préalable et par là-même suffisamment stable (car appartenant au lexique répertorié) pour servir de repère. Ce qui corrobore le fait que as $Q$ est bien le vestige d'une structure corrélative. Le mouvement interne à l'énoncé est en réalité circulaire (as...as).

78 L'énoncé (50), qui est construit à la fois sur as et like, met en évidence le phénomène :

(50) [...] 5. avoidance of substitute words like it, as, for example, 'Where's your milk' instead of 'Where's your milk? Show it to me'

On va, à l'aide de like, de l'ensemble vers le spécimen avec substitute words / it, et 'Where's your milk'...'Where's your milk? Show it to me', avec le support de as, redéfinissent it en s'appuyant sur des phrases obéissant aux règles syntaxiques de la grammaire. (1) est un autre exemple qui illustre autre chose encore, à savoir une validation, par as ... as de l'échantillon posé par alike ${ }^{25}$; ce qui tend à prouver si besoin était que les deux opérateurs acceptent fort bien de "travailler" ensemble :

(1) It is exceptional to find words as alike as meow in English and miaou in French. Meow et "miaou "sont présentés comme faisant partie de la classe des words.Dans le même temps, meow et " miaou" sont des lexies répertoriées, donc stables, acquises. On est bien dans une situation où as ... as entérine la vision de meowet " miaou" comme échantillons, occurrences typiques de words.

81 Ce même énoncé conduit directement à (1') : 
(1') It is exceptional to find words like meow in English and miaou in French.

Enoncé également possible, mais non équivalent. Dans cette version, words est un ensemble de termes dans lequel on trouvera meow et " miaou ". C'est le pluriel sur words qui permet ainsi de jouer sur cet énoncé et les effets de sens de as et like.

Pour ce qui est des mouvements mis en évidence, il semblerait que l'on puisse les retrouver dans les constructions as / like + V. Ce n'est pas là l'objet de l'étude, mais la question mérite d'être posée. J'ai donc demandé à une collègue anglophone de mettre des mots sur la différence de sens, si différence il y avait, qu'elle pouvait ressentir entre She wants to speak English as I do (A) et She wants to speak English like I do (B). Différence de sens il y a, et l'explication fournie est la suivante : en (A) il faut comprendre in the same way as I do (with a particular accent, etc.), tandis qu'en (B) il faut comprendre ... and she wants to do the same ou, selon le contexte, I want to speak English and so does she. On note donc qu'en (A) la glose fait apparaître ce sur quoi se fonde la comparaison, à savoir les propriétés stables correspondant à la notion verbale [speak English]. En (B), il est plutôt question de l'expression d'un rapprochement de deux façons de faire. Par conséquent, il existe bien une différence entre les deux énoncés. Mais ce qui est encore plus intéressant, c'est que d'après cette collègue il faut une pause (orale, ou une virgule) avant like; partant, ce qui suit like est un renchérissement de ce qui précède, après arrêt. La technique énonciative reflète effectivement l'opération mentale : un temps de réflexion est nécessaire à tout procédé de rapprochement de deux choses. En d'autres termes, avec like, un temps de réflexion est nécessaire à tout procédé de construction du repère de la comparaison.

Les lignes qui précèdent ont apporté la matière à partir de laquelle je vais à présent proposer une interprétation du fonctionnement de as et like.

\section{Interprétation}

\subsection{Système des périphrases}

5 Les exemples empruntés à $\mathrm{D}$. Thomas ont permis de mettre en évidence le fait que les énoncés construits sur as et like $(+\mathrm{N})$ sont la représentation d'un mouvement sémantique attaché à chaque type. La relation de comparaison ne se construit pas de la même manière selon qu'elle s'appuie sur as ou like. Q redéfinit, synthétise le contenu sémantique de $\mathrm{P}$ à partir de notions installées avec as, là où $\mathrm{Q}$ apporte une enveloppe formelle et développe le contenu sémantique de $\mathrm{P}$ avec like.

Deux opérations qui peuvent être présentées en termes de périphrases. C'est-à-dire que dans une structure en as, Q est une périphrase substantielle de P (elle vient lui apporter une substance sémantique reconnue), et dans une structure en like, Q est une périphrase formelle de $\mathrm{P}$ (elle vient lui apporter une enveloppe formelle dans laquelle glisser le contenu sémantique du repère, et qui ne vaut que pour ce repère précis dans cet énoncé précis) ${ }^{26}$. Le phénomène est clair dans les énoncés suivants où le mouvement de tous les énoncés en like reflète un procédé de construction de la référence au repère (procédé qui peut s'appuyer sur des participiales ou des relatives, support privilégié d'ajout informationnel) :

(51) He twitched her ringlets and looked at Miss Brodie like a child showing off its tricks. (Spark : 23)

(52) Monica Douglas, later famous for being able to do real mathematics in her head, and for her anger, walked behind them with her dark red face, broad nose, 
and dark pigtails falling from her black hat and legs already shaped like pegs in their black wool stockings. (Spark : 27-28)

(53) She clutched the bars of the grille as if she wanted to escape from the dim parlour beyond, for she was not composed like the other nuns WHO sat, when they received their rare visitors, well back in the darkness with folded hands. (Spark : 35)

(31) It was her habit to come to me every morning to ask what I would have for breakfast, and she spoke like this : “Wud ye have a red herrin? - no ye wudn't. [...].

(54) But now she was giggling openly like a dirty-minded child of an uncultured home. (Spark : 50)

87 La construction $\mathrm{N}$ of $\mathrm{N}$ participe pour beaucoup à l'élaboration du repère, dans la mesure où l'on sait de cette configuration qu'elle pose à plat une relation en pleine élaboration au fil de l'énonciation. La chose se retrouve en (55), où elle est tout à fait en affinité avec la construction verbale to treat somebody like. To treat somebody as existe, mais n'aurait pas été possible ici je pense, à cause de $\mathrm{N}$ of $\mathrm{N}$ :

(55) They treated the new first-formers as if they were not real, but only to be dealt with, like symbols of algebra, and Miss Brodie found this refreshing at first. (Spark: 75)

(56) But the syntactic stage also offers combinations like 'that doggie', 'baby chair' ('Baby's chair'), 'more cereal', which are used [...] (Bolinger : 6)

(57) [...]; reversing the movement of the head, going up instead of down on each accent, in a sentence like 'I will not do it'. (Bolinger : 15)

(58) He starts full of energy but deflates at the end like abagpipe running out of wind. (Bolinger : 32 )

Je terminerai par l'énoncé (17') qui va permettre d'approfondir cette étape interprétative :

(17') Probably all languages use relative " height" as a sign of importance. If in an example like the one just given there happen to be two separations instead of one, it may be necessary to show which is major and which is minor.

Tout se passe comme si en (17') on avait le schéma suivant :

As + entité préconstruite, donc identifiée (ce que pourrait corroborer la structure use $N$ as $N$ dans laquelle il apparaît ici)

Like + entité en construction ou non préconstruite a priori

90 Avec as, il existerait culturellement une entité [a sign of importance] préconstruite ${ }^{27}$, qui ne serait pas sujette à caution. Partant, elle pourrait servir de support stable pour établir une comparaison. As prendrait donc appui sur une entité entérinée au préalable.

91 En revanche, avec like, on n'aurait rien de tel. Like serait le point de départ d'une nouvelle entité. The one just given est parlant. Chaque mot est à sa place et concourt à la construction du sens qui, partant, est progressive. Le repère se crée bien au fil de l'énonciation ou de la lecture.

92 Cet exemple, parmi d'autres, indique clairement que as renvoie à une entité notionnellement bouclée, dépassée; on est dans la notion, son identification est complète. Like pour sa part s'attache à tenter de délimiter les contours de chaque entité pour ce qu'elle vaut à des fins de comparaison à venir dans l'énoncé. Il se différencie ici 
de as pour lequel la comparaison est, sinon contemporaine de l'identification, à tout le moins antérieure, car seule toute chose identifiée est, on le sait, susceptible de servir de support à une comparaison à tout moment. L'identification est en effet la condition sine qua non préalable à la comparaison.

As et like ont donc chacun un rôle qui leur est propre dans le processus d'élaboration de la comparaison. As dit l'identité du repéré avec le repère. Like ne se charge pas de cela. Like n'a pas à se préoccuper de l'identité du repère, ce n'est pas à cela qu'il s'attache. Puisque c'est le rôle de as, like est réservé à l'expression de la relation entre les entités. Like porte sur la nature du lien entre les entités et non la nature de ces entités. Il est occupé à indiquer que les entités sont différentes en essence et ne pourront jamais se superposer. Il assied leurs différences notionnelles. Elles valent par leurs différences, par ce qui les oppose et non pas réellement par ce qui les rapproche, comme cela a été dit, car cela se baserait sur une propriété commune. Les entités repérées par like valent par leurs différences et non par leurs points communs. Cette opposition bloque tout rapprochement total, en pointant sur les dissimilitudes. C'est la raison pour laquelle like ne peut véhiculer l'idée d'une correspondance parfaite, d'une identité parfaite. Tout au plus est-il donc capable d'indiquer une similarité, aussi forte soit-elle.

Et c'est précisément ce qui a pu conduire à la catégorisation de as / like en termes d'identification / différenciation, et leur rapprochement avec be et have qui me semble tout naturel ${ }^{28}$. Il est possible de pousser plus avant le rapprochement en le présentant en termes d'identification / localisation.

\subsection{Le système identification / localisation}

L'exemple (17') conduit à une nouvelle formulation du phénomène as / like. Il est en effet légitime de penser que l'opposition as / like en anglais rejoue l'opposition be / have où deux systèmes fonctionneraient sur des bases similaires. A savoir :

\begin{tabular}{|c|c|c|c|}
\hline LIKE / & AS & HAVE / & BE \\
\hline $\begin{array}{l}\text { différences } \\
\text { opérateur } \\
\text { localisation }\end{array}$ & $\begin{array}{l}\text { identité } \\
\text { opérateur } \\
\text { d'identification }\end{array}$ & $\begin{array}{l}\text { différences } \\
\text { opérateur } \\
\text { localisation }\end{array}$ & $\begin{array}{l}\text { identité } \\
\text { opérateur } \\
\text { d'identification }\end{array}$ \\
\hline
\end{tabular}

Like, opérateur de localisation, met en relation deux éléments distincts avec intégration $\mathrm{du}$ second dans la sphère du premier. Ici, la localisation consiste en outre en une opération d'opposition entre les deux éléments en question. Ils sont confrontés sans jamais pouvoir se confondre, ou être confondus. $X$ like $Y=X$ has the characteristics of $Y$ (but will never be $Y$ ).

97 As, opérateur d'identification fonctionne pour sa part comme un hub. En effet, la nature identitaire de as fait qu'il est stable, et lui permet donc de servir de support à un nouveau sous-système, celui qu'il forme avec so. Dans les constructions as $\mathrm{p}$, so $\mathrm{q}{ }^{29}$, $\mathrm{q}$ dépend de $\mathrm{p}$. Je pense que l'on peut dire que q est localisé par rapport à $p$ dans la mesure où $q$ apparaît comme une conséquence de $\mathrm{p}$, conséquence qui n'est donc pas $\mathrm{p}$. La différence entre like et so se situerait dans la nature même de la localisation qu'ils opèreraient. On a vu que like s'attache à souligner les différences à l'inverse de ce que fait as. So s'attacherait lui à 
souligner les ressemblances posées par as. Il rapprocherait l'entité repérée au maximum de l'entité repère, sans qu'il y ait contact. Dans le cas où il y a contact, on a la structure as ... as. D'où le schéma :

\begin{tabular}{|l|l|l|}
\hline Like / & as / & so \\
\hline Localisation & identification & localisation \\
\hline
\end{tabular}

Sur le plan sémiologique, on peut retrouver un écho de ce qui vient d'être dit, en reconnaissant le poids tout relatif de la remarque. As et so ont en commun la sifflante $-s$, symbole de continuité. Continuité de la perception de la chose référée. As et so tendent vers l'identité. Like comporte une occlusive vélaire, qui va effectuer un blocage complet de l'air à un certain point du palais. Like dit le hiatus dont il est la trace dans l'élaboration de la comparaison.

L'identification se fait donc plus explicite quand on a le tour as ... as :

(59) To make a sentence a child must recognize certain things as the same and certain things as different, must have names for them, ad must combine the names. (Bolinger : 5) ${ }^{30}$

The same développe ici as. The same dit l'identité véhiculée par as ${ }^{31}$. On est à une étape intermédiaire entre as $+N$ et as being $N$.

C'est encore l'identification qui va permettre d'expliquer que l'on a des tours en except as mais pas en except like :

(4) Nobody in her life, at home or at school, had ever spoken of Calvinism except as a joke that had once been taken seriously.

Nous savons en effet que la négation ne peut reposer que sur du positif, préalablement identifié. La négation est subordonnée à l'affirmation positive de l'existence. De même, j'ai dit à propos de mon énoncé (1) :

(1) It is exceptional to find words as alike as meow in English and miaou in French. qu'il illustre, avec as alike as, "une validation par as ... as de l'échantillon posé par alike", je peux à présent poursuivre et dire: “(1) illustre une validation par as ... as, comme chose acquise,de l'échantillon posé par alike".

104 Mais là n'est pas tout ce que l'on peut dire de as et like. Un troisième groupe d'énoncés fait ressortir la subjectivité associée à like, dont il a déjà été implicitement question. Les énoncés qui viennent vont ainsi permettre d'approfondir le principe même de la préconstruction.

\section{De la préconstruction vers le principe de la préconstruction énonciative}

Dans les exemples qui suivent, la référence au repère de la comparaison se teinte d'une (forte) subjectivité :

(60) - walking like Sybil Thorndike, ma'am. (Spark: 23)

(61) The outsides of Old Edinburgh churches frightened her, they were of such dark stone, like presences almost the colour of the Castle rock, and were built so warningly with upraised fingers. (Spark:35) 
(62) Miss Brodie had shown them a picture of Cologne Cathedral, like a wedding cake, which looked as if it had been built for pleasure and festivities, and parties given by the Prodigal Son in his early career. (Spark : 35 )

(63) They were therefore obliged to compete with each other in every walk of life within the school and on the wind-swept hockey fields which lay like the graves of the martyrs exposed to the weather in an outer suburb. (Spark :78)

106 meilleure référence possible.

(64) 'Rose', said Miss Brodie, 'is like a heroine from a novel by D. H. Lawrence.' (Spark : 110)

107 Je terminerai par l'étude de l'échange suivant :

(65) 'Why, it's like Miss Brodie !' said Sandy.

'It's terribly like Miss Brodie.'

'Though, of course it's Rose, it's more like Rose, it's terribly like Rose.'

It still looked like Miss Brodie.

- [...] ; it was the type of stare from Rose's blue eyes, perhaps, which was like the dominating stare from Miss Brodie's brown.

- The portrait was very like Miss Brodie. (Spark : 99) ${ }^{32}$

ici de l'observation d'un portrait peint. It désigne donc cette peinture, d'un physique, d'une “enveloppe extérieure", pour reprendre cette appellation utilisée plus haut. La relation de comparaison dont l'extrait se fait l'écho naît ici de la vision de l'énonciateur et de son interprétation de l'œuvre. Elle est par la force des choses toute subjective. (65) est une saisie " sur le vif". Nous sommes dans l'instant, en cours de travail de construction de la référence à la chose que l'on veut élever au rang de repère, en T0. Avec like, l'énonciateur est explicitement présent.

Voyons encore :

(66) He's got absolutely no claim on me at all, and yet a large-sized chunk of my existence seems to be spent in fussing over him like a bally old hen and hauling him out of the soup. (Wodehouse : 98)

(67) At the end of the second refrain the entire house was shouting for an encore, and the kid with the voice like a slate-pencil took a deep breath and started to let it go once more. (Wodehouse : 160)

Le support sur lequel se crée la comparaison est propre à l'énonciateur. En (67) c'est l'énonciateur qui décide de qualifier la voix de la manière dont il le fait, c'est lui qui choisit les mots qu'il utilise, et personne d'autre à sa place. Partant, on est en droit de se demander à qui véritablement attribuer as, à qui véritablement attribuer like. Se pourraitil donc que le fonctionnement et les conditions d'emploi de as et de like soient liés à ce que l'on pourrait appeler la "préconstruction énonciative", c'est-à-dire la préconstruction propre à l'énonciateur?

111 Charles Bally a exprimé quelque chose d'approchant en disant :

Tel fait de langage exprime-t-il surtout une idée ou surtout un sentiment?

La plupart du temps, nous n'énonçons pas des idées et des jugements sur les choses,

mais les impressions que ces choses font sur nous. (Bally $1909: 152)^{33}$

112 Il semble légitime que l'on puisse alors penser que chaque énonciateur aurait un préconstruit personnel qui influencerait sa vision, et par conséquent sa manière de rendre, la comparaison. 


\subsection{Différence du préconstruit}

113 (66) illustre très bien ce que j'entends par “différence du préconstruit". Tout comme il permet de théoriser cette différence. A partir de cet énoncé, on se rend compte que as et like ne reflètent pas le même préconstruit.

Like introduit un préconstruit personnel, subjectif. Like rejoint bien la localisation car avec like, chaque entité participe à part entière au sémantisme final. Chaque entité doit être distinguée et distinguable. As n'y serait pas possible. Car ce qu'introduit as, c'est un préconstruit collectif, partagé (propre à un groupe social, culturel, professionnel, etc.). En ce sens, as rejoint bien l'identification, car avec as, le préconstruit collectif doit être stable pour le groupe. Sa stabilité est garante d'une reconnaissance et d'une acquisition par le plus grand nombre pour pouvoir, par la suite, être reprise et servir de repère.

La raison profonde pour laquelle as et like véhiculent chacun un préconstruit pourrait tout simplement correspondre aux deux protagonistes de la relation de communication. Ce préconstruit n'appartiendrait pas tant uniquement à l'énonciateur mais s'adresserait en priorité au co-énonciateur.

116 En effet, il semble évident qu'il existe une nécessité d'explicitation de la relation pour le co-énonciateur. Pour lui faciliter la compréhension, pour lui fournir les éléments suffisants et nécessaires afin qu'il puisse, à son tour, reconstruire l'entité telle qu'elle est conçue par l'énonciateur.

117 Avec as, la construction de la comparaison serait minimale car elle reposerait sur un préconstruit collectif. Avec like, la construction de la comparaison serait maximale, car elle correspondrait à la propre perception de l'énonciateur. Le préconstruit du coénonciateur étant par définition le sien, il est à l'évidence différent de celui de l'énonciateur. Lui donner les éléments de la comparaison lui évite de faire porter son préconstruit individuel sur la chose référée, de la voir avec les notions qui sont les siennes. L'énonciateur, avec like, forcerait la compréhension de la comparaison (telle qu'il l'entend lui) de la part du co-énonciateur.

118 De fait, quand je parle, je sais ce que je dis (dans une situation normale). Je ne sais pas ce que je dis dans une situation anormale (troubles du langage, de la parole, affection mentale...), ou je suis ainsi étiquetée ("elle ne sait pas ce qu'elle dit / raconte") quand mon co-énonciateur ne me comprend pas.

119 C'est le co-énonciateur qui conclut de la sorte dès lors qu'il ne peut interpréter, décoder, le message. Ainsi, tout le travail linguistique en amont de l'énonciation a non pas tant pour but de refléter le comportement de l'énonciateur que d'être, aussi, une démarche pédagogique à l'intention du co-énonciateur, une pédagogie langagière, une pédagogie communicante.

120 Le principe distributionnel caractérisant as et like fonctionne, même dans des cas où cela ne va pas de soi a priori.

\subsection{Discussion de contre-exemples (apparents)}

121 Des éléments du contexte qui peuvent sembler insignifiants appuient l'interprétation présentée dans ces lignes. Les énoncés (68), (69), (70) et (71) en sont la juste illustration. 
(68) The first effect on Sandy was an adverse one, for she had been on the point of obtaining permission to go for walks alone in such just isolated spots as that in which Jenny's encounter had taken place. (Spark: 67) l'ensemble vers l'échantillon, il y a localisation du terme échantillon dans la sphère de l'ensemble. Ce, bien que l'énoncé s'appuie sur le code civil, c'est-à-dire sur un préconstruit partagé et qui correspond aux propriétés de as. Car ce préconstruit partagé, collectif de (69) est inclus dans un ensemble plus vaste, qui le dépasse, et dont il n'est plus qu'une partie. The legal code est une illustration de formalized systems, il ne définit pas formalized systems dans son intégralité, il ne permet pas d'identifier formalized systems.

(70) Sometimes those parts are pretty well standardized, like the suffix-ness or the prefix un- . (Bolinger : 54 )

(71) Trade names make easy use of almost any fragment like the -roni of macaroni that is reattached in Rice-a-Roni and Noodle-Roni. (Bolinger : 54)

(70) est un formidable contre-exemple à première vue, car the suffix -ness et the prefix un - renvoient à des entités répertoriées ${ }^{34}$. Nous sommes donc dans le domaine de as. Et pourtant nous avons like. Il s'avère en fait que l'on retrouve ce mouvement de l'ensemble vers l'échantillon propre à like. De même, en (71), où -roni est un suffixe répertorié. Pourtant on assiste encore à la construction de l'exemple pour l'énoncé précisément (l'apport sémantique se construit de gauche à droite : the .. of ...), et on a like.

Car ce qui se passe en (70) et (71), c'est que les choses introduites par like ne se suffisent pas à elles-mêmes pour attribuer une nouvelle identité. Elles ne sont que partie, unique qui plus est, d'une identité plus grande. En effet, -ness n'est qu'un suffixe parmi d'autres. Tout comme un- n'est qu'un préfixe parmi d'autres. Like va venir dans et pour l'énoncé introduire un maillon qui contribue à la construction, par la suite, d'une entité en $\boldsymbol{a} \mathbf{s}^{35}$. L'énonciateur a besoin des échantillons -ness / un- pour parvenir à circonscrire les entités suffixes / préfixes.

Nous aurions sans doute pu avoir like a suffix ; as -ness, as un-, voire a suffix such as -ness, a prefixsuch as un-,-roni as in macaroni. Mais pas * like the suffix. Le déterminant appelle effectivement un complément d'information qui, avec like, ne peut être introduit qu'à sa suite. Il faut que ce qu'introduit like soit précisé au maximum. Et cette précision suit toujours like dans la châne linéaire que constitue toute phrase. 


\section{Conclusion}

127 J'ai tenté dans ces lignes de réfléchir autrement à la manière dont as et like s'organisent dans l'élaboration de l'expression de la comparaison dans la langue anglaise. En m'appuyant sur les principes de l'identification et de la localisation que, dans le même temps, j'ai souhaité définir clairement, j'ai essayé de montrer que les deux opérateurs sont en réalité à la base d'un système reposant sur la préconstruction. Une préconstruction "collective" pour as et "personnelle" pour like. Une préconstruction qui, en outre, ne serait pas tant notionnelle que ce que l'on pourrait appeler “énonciative ", et qui ne concernerait pas uniquement l'énonciateur, mais également le co-énonciateur. Qui serait même peut-être uniquement destinée au co-énonciateur.

\section{BIBLIOGRAPHIE}

ADAMCZEWSKI, H. (1982). Grammaire linguistique de l'anglais, Paris : Armand Colin, 354 p.

BALLY C. (1909). Traité de stylistique française, Georg \& Cie S.A.

BOLINGER, D. (1968). Aspects of Language, New York : Harcourt, 326 p.

Collins Cobuild English Language Dictionary (1992). Glasgow : Harper Collins Manufacturing.

FLUCHA, L. (2000). “ AS dans les constructions du type $X+A S+$ sujet + Prédicat”, in Syntaxe \& Sémantique, 1, Caen : Presses Universitaires de Caen, 157-182

GILBERT, E. (1998). “Quelques remarques sur AS et la construction des valeurs référentielles ”, in Travaux Linguistiques du CERLICO, $\mathrm{n}^{\circ} 11$, Rennes : Presses Universitaires de Rennes, 103-125

GUILLAUME, G. (1919). Le problème de l'article et sa solution dans la langue française, Paris : Nizet.

GUIMIER, C. (1997). “Présence ou absence du sujet ou d'un complément essentiel dans certaines propositions introduites par AS ", in Travaux Linguistiques du CERLICO, $\mathrm{n}^{\circ} 10$, Rennes : Presses Universitaires de Rennes, 205-230

JOLY, A. et O'KELLY D. (1990). Grammaire systématique de l'anglais, Paris : Nathan, 495 p.

LAB, F. (1999). "Is as like like or does like look like as ?", in Les Opérations de Détermination, Quantification / Qualification, Paris : Ophrys, 83-100

LAPAIRE J.-R. \& ROTGÈ W. (1991). Linguistique et grammaire de l'anglais, Toulouse : Presses Universitaires du Mirail, $734 \mathrm{p}$.

LAROUSSE (1994). Le Dictionnaire pratique du français, Paris : Larousse, $542 \mathrm{p}$.

LARREYA, P. (1996). “ On the Semantics of As and So ”, SIGMA, 17-18, 97-130.

LE GOFFIC P. (1991). “Comme, adverbe connecteur intégratif : éléments pour une description ", in Travaux Linguistiques du CERLICO, $\mathrm{n}^{\circ} 4$, Rennes : Presses Universitaires de Rennes, 11-31 
LE GUAY, E. (1995). Recherches sur le Past Perfect en anglais contemporain, Thèse, Université Paris IV, $430 \mathrm{p}$.

QUIRK R., GREENBAUM S., LEECH G. N. \& SVARTVIK J. (1985). A Comprehensive Grammar of the English Language, Londres : Longman, $1779 \mathrm{p}$.

ROCHETTI, A. (1980). “ De l'Indo-Européen aux langues romanes : une hypothèse sur l'évolution du système verbal ", in Langage et psychomécanique du langage pour Roch Valin, Joly, A. et Hirtle, W., H. (dir.). Lille : Presses Universitaires de Lille.

ROUSSEL E. (1999). " She had been healthy and beautiful when he married her ",in Anglophonia, 6, Toulouse : Presses Universitaires du Mirail, 235-258.

ROUSSEL E. (2003). “When et la hiérarchie propositionnelle ”, in : CORELA - Cognition, Représentation, Langages, 1, http://revue-corela.org. 16 p.

ROUSSEL E. (2003). “ Le commentaire associé au passif anglais ”, Cahiers CRISCO, n¹5, Université de Caen.

ROUSSEL E. (2003). “ Remarques sur la construction de la valeur de commentaire véhiculée par le passif anglais", en soumission

SPARK, M. (1961). The Prime of Miss Jean Brodie, London : Penguin.

SWEET, H. (1891-1898). A New English Grammar, Part I, Londres : Oxford University Press, 519 p.

The Oxford Dictionary of English Etymology (1992). Oxford : Oxford University press.

THOMAS, D. (1954). Under Milk Wood, London : Everyman Classics

WODEHOUSE, P. G. (1923). The Inimitable Jeeves, Vintage, 1991.

\section{NOTES}

1. J'adresse tous mes remerciements à Claude Guimier pour sa lecture critique de ce travail et la pertinence de ses remarques.

2. J'ai intentionnellement restreint le champ de mon étude, avant tout par intérêt personnel.

3. P. Larreya, par exemple, propose une définition approchante: “Comparer, c'est affirmer l'existence de similitudes ou de différences." (Grammaire explicative de l'anglais, p. 335)

4. Bien que j'utilise ici les termes de "quantité " et de "qualité", ceux-ci ne sont pas à prendre au sens qui leur est actuellement attribué dans la Théorie des Opérations Enonciatives. Tout en empruntant quelques concepts largement répandus de cette théorie, je ne la maîtrise pas et ne saurais prétendre travailler dans son cadre.

5. C'est moi qui souligne. Ceci est dû à l'origine de "comme" (rattaché à "commode " (= "à la bonne mesure ", " approprié "), lui-même issu de la famille de "moule" et tout ce qui traite de la mesure, de la juste mesure, de la manière, du genre (lat. modulus: "mesure"). Le Petit Robert : 1681.

6. Critère qui se retrouve dans l'origine de "comme ", où il est question non pas de l'essence de la chose, mais de son évaluation.

7. Mais dans des emplois figés... J'ai en revanche trouvé une occurrence de as alike as : (1) It is exceptional to find words as alike as meow in English and miaou in French. (Bolinger $1968: 15$ )

8. Pour ce dernier exemple, la période de la fin de son acceptabilité se situe nous l'avons vu entre les XIVe et XVIIIe siècles. A partir de ce moment-là, c'est le tour like as qui est utilisé. Ensuite les opérateurs se dissocient et les emplois se particularisent, as se spécialisant dans l'usage littéraire et like dans l'usage informel. Nous avons dans notre corpus un énoncé révélateur à ce sujet (on 
laissera de côté la présence de if ici) : (2) 'She looks really like as if she won't have any prime,' Sandy said. 'The word 'like' is redundant in that sentence.'(Spark, 1961: 92)

9. (3) The main problem with such vast quantities is to find not resemblances but differences, to make a given combination of sounds sufficiently unlike every other combination so that no two will be mistaken for each other. (Bolinger : 16).

10. (4) Nobody in her life, at home or at school, had ever spoken of Calvinism except as a joke that had once been taken seriously. (Spark: 108). (5) 'Neither she is,' said Sandy,'except as a side interest. She's a born Fascist, have you thought of that?' (Spark : 125). (6) We are unable to use any other medium except as an accidental help.(Bolinger : 13).

11. (7) Sandy was sometimes embarrased by her mother being English and calling her 'darling', not like the mothers of Edinburgh who said dear'. (Spark : 18).

12. The Oxford Dictionary of English Etymology, p. 528. C. Guimier souligne le sens souvent associatif de ge-, comme dans gefera (" compagnon de voyage"), gebedela (" concubin "), gesey (pluriel de scol, " paire de chaussures"). Dans ge-lice, ge- marque le rapprochement de deux objets déclarés avoir la même forme (lice).

13. Lician, verbe, existe en Vieil-Anglais, et serait à l'origine du verbe like. (c. Guimier).

14. Collins Cobuild English Language Dictionary et The Oxford Dictionary of English Etymology.

15. Vers 1100-1400, c'est-à-dire à la période même où apparaît ilich-e / ilik-e.

16. Henry Sweet, A New English Grammar, p. 436.

17. Sens le plus couramment admis, je n'en fais pas le sens central de as.

18. Je renvoie à ce propos à l'énoncé (2).

19. Voir 2.2. Dans le même cadre théorique, on lira avec intérêt l'article d'Eric Gilbert (1998) sur as : "Quelques remarques sur As et la construction des valeurs référentielles".

20. Une remarque comparable est faite par Adamczewski (1982: 269): “As signale que l'on constitue une identité entre deux domaines (événements, temps, manières, etc.) [...] As s'oppose à like dans ce rôle. As reste à un niveau proche de l'extralinguistique ; like s'en éloigne : He is dressed as a clown (il tient effectivement le rôle d'un clown) He is dressed like a clown (il ne s'agit pas d'un clown, mais de quelqu'un qui est habillé en dépit du bon sens) Adamczewski 1982 : 269.On ne peut s'empêcher de poursuivre en pensant, à propos du premier exemple: “avec tout ce que cela suppose, notamment que l'on sache ce qu'est et comment s'habille un clown " et, à propos du second : "on a là un bel exemple de vision subjective ".

21. La citation est de Robert D. Wilson (" A Criticism of Distinctive Feature ", Journal of Linguistics, 2, 201, 1966)

22. Je concède que (26) n'est pas strictement conforme au schéma as $+\mathrm{N}$, mais je le cite car je pense qu'il est gouverné par le même principe.

23. Le déterminant the est ici décisif dans l'instauration du caractère unique de l'entité étalon référée.

24. Terme qui, en vieux français, désignait un "étalon de mesure " (1636). Le Petit Robert: 819.

25. Alike ayant par la suite donné like. C'est donc comme si l'on avait as like as, qui existe (voir supra As like as two peas).

26. Enonciativement, cela signifie que l'énonciateur s'implique moins avec as qu'il ne le fait avec like.

27. Toute la structure $\mathrm{N}$ of $\mathrm{N}$ serait ici préconstruite; il n'est pas question dans cet énoncé de mettre à plat la relation entre sign et importance. C'est l'entité tout entière qui est présentée comme susceptible de s'opposer à une autre entité qui serait par exemple a sign of smallness.

28. Je travaille depuis quelque temps déjà sur ces principes (Roussel 1999, 2003, et à paraître).

29. Où l'on n'omettra pas de donner tout son sens à la virgule. Je pense qu'il s'agit du même que celui vu avec like $+V$, car avec so il y a mise en mots, effort de dire une relation de cause à conséquence qui ne va pas de soi, puisqu'elle nécessite le support des opérateurs.

30. Nous avons ici deux constructions en as parallèles. 
31. Et different la nie.

32. Like est en gras dans le texte lui-même.

33. Souligné dans le texte original.

34. A la vue de cet exemple, je concède à $C$. Guimier que la notion de "répertorié ", qui " n'est qu'une notion linguistique" pour reprendre ses termes, n'est peut-être pas la meilleure pour caractériser as. Peut-être pourrait-on parler d'une identification qui n'est plus à faire.

35. C'est-à-dire que nous pourrons par la suite avoir la construction des références suivantes: the prefix un-puis as the prefix un-(dans cet ordre), the suffix-ness puis as the suffix-ness.

\section{RÉSUMÉS}

As et like sont des marqueurs privilégiés de l'expression de la comparaison en anglais et nombre de travaux ont été effectués à leur sujet. Mon but ici est de tenter de montrer que, comme d'autres dans la langue anglaise, ces marqueurs forment un système et que, en tant que système, ils se répartissent les rôles de manière claire et précise, notamment lorsqu'il est question de l'expression d'une comparaison construite sur le modèle : as/like +dét. + N. Je serai ainsi amenée à travailler à nouveau les principes d'identification et de différenciation qui sous-tendent le fonctionnement de as et de like et qui, à mon sens, reposent non pas sur un phénomène de préconstruction unique mais bien sur un type de préconstruction propre à chacun des marqueurs. D'où cette approche en termes de " répartition des rôles".

As and like are privileged markers as far as the expression of comparison in English is concerned. They have been the subject of numerous works. Here I aim to show that these markers make up a new system in the English language. As such, each of them has a specific function as soon as comparison (following the structure as/like det. $N$ ) is to be expressed. Consequently I am going to study the principles of equation and location again. Indeed these two principles underlie the way as and like operate and I think that they rest on preconstruction. Not preconstruction in the accepted sense of the word, but a kind of preconstruction proper to each marker.

\section{INDEX}

Mots-clés : as, like, comparaison, mouvement, échantillon, synthèse, développement, périphrase, préconstruction énonciative

\section{AUTEUR}

EMMANUELLE ROUSSEL

Université de Caen - CRISCO (UMR 6170) 